\title{
MICROFABRICATED WORKBENCH DESIGNED TO INVESTIGATE THERMOELECTRIC PROPERTIES OF LOW-DIMENSIONAL MATERIALS
}

\author{
DukSoo Kim ${ }^{1,}$ James Kally $^{2}$, Nitin Samarth ${ }^{3,4}$, and Srinivas Tadigadapa ${ }^{1,4}$
}

${ }^{1}$ Department of Electrical Engineering, The Pennsylvania State University, University Park, USA

${ }^{2}$ Department of Materials Science and Engineering, The Pennsylvania State University, University Park, USA

${ }^{3}$ Department of Physics, The Pennsylvania State University, University Park, USA

${ }^{4}$ Materials Research Institute, The Pennsylvania State University, University Park, USA

\begin{abstract}
Microfabricated workbench has been developed to investigate thermoelectric properties of low-dimensional materials. The central area of the chip has high density of test sites for improving the probability of landing single nanowire or nanotube at desired position on the workbench when it is drop cast from solution. This obviates the extensive effort of nanomaterial manipulation required for measurement and provides a reliable platform to measure the thermal, thermoelectric, and electrical characterization across various nanomaterials. To demonstrate the efficacy of the device, the three thermal and electrical transport properties and thermoelectric figure of merit $\mathrm{ZT}$ of $\mathrm{GaAs} / \mathrm{MnAs}$ core/shell nanowire were successfully measured using the workbench at temperatures ranging from $150 \mathrm{~K}$ to $300 \mathrm{~K}$.
\end{abstract}

\section{INTRODUCTION}

Low-dimensional materials (LDMs), such as nanoribbons, nanowires, and nanotubes, have attracted great attention as thermoelectric materials due to their potential for having improved thermoelectric efficiency [1,2]. However, the lack of a thermal equivalent of an "ammeter" makes the measurement of thermal and thermoelectric properties (thermal conductivity and Seebeck coefficient) in LDMs challenging. Although thermal workbenches have been reported earlier, these devices require considerable effort in terms of placement and alignment of the LDMs on the workbench or are dedicated for measurement of a single material and cannot be used for a variety of LDMs [3, 4]. In this work, we have designed and fabricated micro-workbenches with high surface density of test-sites in the central area of the chip to enhance the probability of landing a single LDM at the desired positions without any physical manipulation when drop casting from a solution containing the LDMs.

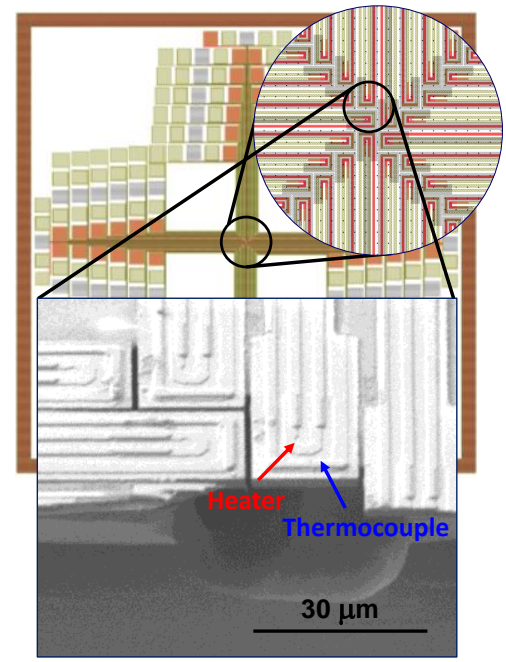

Figure 1: Mask overview of the overall workbench chip. Insets show the zoom-in of the workbenches and an oblique view SEM of the fabricated workbench showing the undercut tip areas.

9781940470016/HH2014/\$25C2014TRF
The workbench consists of several closely placed thermally isolated platforms (Figure 1). Each platform has an integrated heater, thermocouple temperature sensor and platinum contact pad. The heater and thermocouple junction are placed at tip of the thermally isolated and freestanding part of the platform. The platinum pad is on top of platform and provides electrical connection to the LDM and is electrically isolated from the thermocouple and heater. 60 platform tips are located in $250 \mu \mathrm{m} \times 250 \mu \mathrm{m}$ central area of the workbench for improving the probability of landing a single LDM bridging two neighboring platform tips when solution containing materials is drop cast. Neighboring platform tips are $1 \mu \mathrm{m}$ apart from each other (inset, Figure 1). Polysilicon-gold thermocouple is used for high-sensitivity temperature measurements.

\section{FABRICATION PROCESS}

The fabrication process is shown in Figure 2. First, silicon nitride $(0.3 \mu \mathrm{m}) /$ silicon oxide $(1.4 \mu \mathrm{m}) /$ silicon nitride $(0.3 \mu \mathrm{m})$ is deposited on silicon wafer as a stress-compensated stack using low pressure chemical vapor deposition (LPCVD) and deposition of polysilicon (LPCVD, $0.4 \mu \mathrm{m}$, boron-doped, $10^{19} \mathrm{~cm}^{-3}$ ) is followed. The polysilicon layer is patterned to define heater and one leg of thermocouple using photolithography and reactive ion etching (RIE). And titanium $(20 \mathrm{~nm}) /$ gold $(400 \mathrm{~nm})$ layer is deposited and patterned using lift-off process to complete the thermocouple structure (Figure 2(a)). Afterward, aluminium oxide (20 $\mathrm{nm}) /$ hafnium oxide $(30 \mathrm{~nm})$ is deposited using atomic layer deposition (ALD), which electrically insulates heater and thermocouple from the following titanium $(30 \mathrm{~nm})$ /platinum (100 $\mathrm{nm}) /$ nickel $(150 \mathrm{~nm}$ ) layer patterned using lift-off process (Figure 2(b)). RIE and deep reactive ion etching (DRIE) then are performed to etch the ALD layer, stress-compensated stack and silicon substrate using nickel layer and photoresist as a mask. Lastly, the silicon substrate is etched in an isotropic manner by xenon

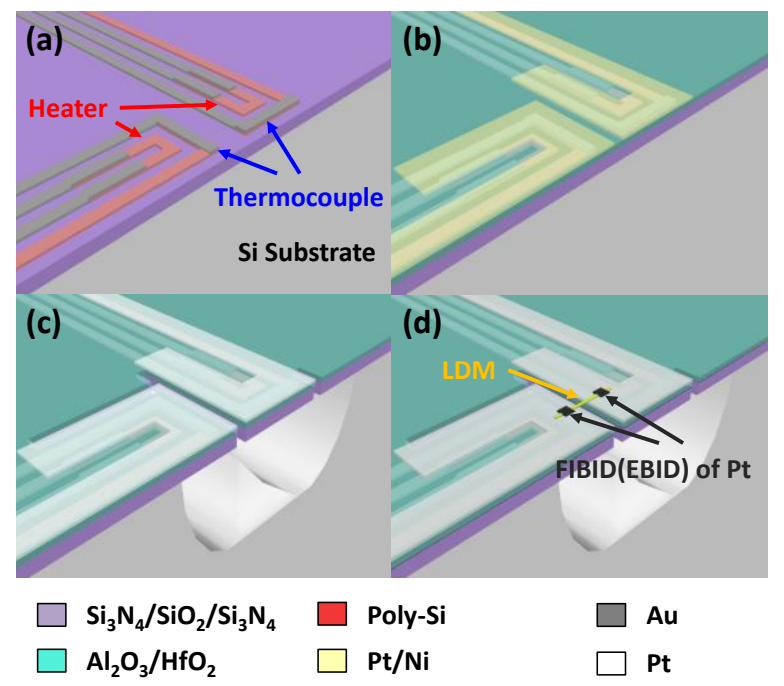

Figure 2: Schematic diagram of fabrication process.

Solid-State Sensors, Actuators and Microsystems Workshop Hilton Head Island, South Carolina, June 8-12, 2014 
difluoride etching and wet etching of nickel is followed (Figure 1(c)). Placement of the LDM on workbench is achieved by drop casting from solution. And focused ion beam (FIBID) or electron beam induced deposition (EBID) of platinum is used to provide mechanical anchors and good electrical and thermal contact between the material and platinum pads of workbench (Figure 1(d)).

\section{CALIBRATION OF THERMOCOUPLE}

Polysilicon-gold thermocouple was made for measuring the temperature of platform tips. However, a calibration is necessary for extracting temperature rise from the thermocouple voltage. For such calibration, the workbench has a special structure where two platform tips connected to each other through a $2 \mu \mathrm{m}$-wide bridge consisting of stress-compensated stack/ALD/titanium/platinum layers (insect, Figure 3(a)) and was used as follows.

Seebeck coefficient at $300 \mathrm{~K}$ was obtained using heater, on same platform with thermocouple, as a thermistor. Initially, resistance of heater was measured with small current $( \pm 20 \mu \mathrm{A})$ using Keithley 2400 at a temperature of $310 \mathrm{~K}$. Thereafter, resistance of heater and thermocouple voltage were simultaeously measured, at an ambient temperature of $300 \mathrm{~K}$, while sweeping current through the heater from $20 \mu \mathrm{A}$ to $600 \mu \mathrm{A}$. Keithley $2182 \mathrm{~A}$ nanovoltmeter was used to measure the thermocouple voltage. Figure 3(a) shows resistance of heater and thermocouple voltage as a function of heating power. By matching the resistance of the heater corresponding to that obtained at $310 \mathrm{~K}$, the thermocouple voltage at $310 \mathrm{~K}$ was obtained. The Seebeck coefficient of thermocouple would be calculated by dividing the extracted thermocouple voltage with temperature rise of thermocouple junction. COMSOL finite element model (FEM) simulation was performed to check the temperature difference between heater and thermocouple junction. It was determined that the temperature rise

(a)
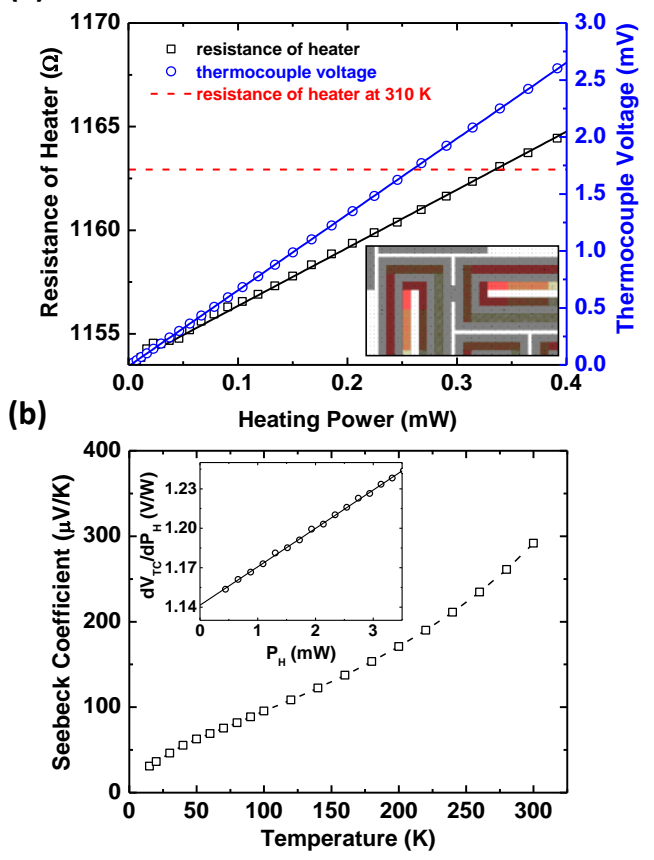

Figure 3: (a) Resistance of heater and thermocouple voltage as a function of heating power at $300 \mathrm{~K}(\mathrm{~b})$ Seebeck coefficient of polysilicon-gold thermocouple as a function of temperature. Inset shows derivative of thermocouple voltage with respect to heating power as a function of heating power at $280 \mathrm{~K}$ ( $V_{T C}$ : thermocouple voltage, $P_{H}$ : heating power). of the thermocouple junction is $24.1 \%$ smaller than that of heater which allowed accurate calibration of the thermocouple junction from that of heater.

The Seebeck coefficient at lower temperatures was obtained using the value at $300 \mathrm{~K}$. If it is supposed that Seebeck coefficient changes linearly with respect to temperature and temperature of thermocouple junction changes linearly according to heating power, thermocouple voltage can be expressed as

$$
\begin{gathered}
S(T+\Delta T)=S(T)+a \Delta T \\
V_{T C}=\int_{0}^{\Delta T} S\left(T+\Delta T^{\prime}\right) d \Delta T^{\prime}=S(T) \Delta T+\frac{a}{2} \Delta T^{2} \\
=S(T) b P_{H}+\frac{a b^{2}}{2} P_{H}{ }^{2}
\end{gathered}
$$

where $S$ is Seebeck coefficient, $T$ is the temperature of cryostat, $\Delta T$ is temperature rise of thermocouple junction, $P_{H}$ is heating power, $a$ is the rate of change of Seebeck coefficient with respect to temperature, and $b$ is a ratio of temperature rise of thermocouple junction to heating power. Differentiating equation (2) gives

$$
\frac{\partial V_{T C}}{\partial P_{H}}=S(T) b+a b^{2} P_{H}
$$

Inset of Figure 3(b) shows the derivative of thermocouple voltage with respect to heating power as a function of heating power at the cryostat temperature of $280 \mathrm{~K}$. In this case we used the heater of neighboring platform that is thermally connected, through a bridge, to the platform with thermocouple. We found that using heater and thermocouple of the same platform resulted in a noisy derivative of thermocouple voltage. Equation (1) and (3) allow us to calculate $S(280)$ using the intercept and the slope of inset of Figure 3(b) and known value of $S(300)$. The Seebeck coefficient of thermocouple was obtained using this method at temperatures ranging from $300 \mathrm{~K}$ down to $15 \mathrm{~K}$ (Figure 3(b))

\section{MEASURING THERMOELECTRIC PROPERTIES OF LOW-DIMENSIONAL MATERIALS}

The workbench on which LDM was anchored as explained previously is placed in a vacuum cryostat with pressure below $5 \times 10^{-6}$ Torr to minimize the effect of parasitic heat transport through ambient air. And a sinusoidal voltage with angular frequency $\omega$ is applied to polysilicon heater of one platform using Keithley 3390 function generator and it in turn produces $2 \omega$ temperature fluctuation. Temperature rise of heated or neighboring platform tip is obtained by measuring $2 \oplus$ voltage of polysilicon-gold thermocouple using SR 830 lock-in amplifier. And $2 \omega$ voltage between two platinum pads is also measured using lock-in amplifier, which represents thermoelectric voltage induced in LDM-platinum thermoelectric circuit. The amount of heat dissipated in the heater (heating power) is calculated by measuring applied voltage and current to the heater using Keithley 2001 and Keithley 2000 multimeters.

\section{Thermal Conductivity}

Initially, the thermal conductivity of LDM at $300 \mathrm{~K}$ is extracted by matching FEM simulation to measured heating power and temperature rise of both thermocouple junctions. For FEM simulations, bulk values of thermal conductivity of gold and silicon were used and the thermal conductivity of platinum was extracted using Wiedemann-Franz law from measured electrical conductivity. The thermal conductivity of rest of the materials was obtained from reference papers [5-9]. However, FEM cannot be used at low temperatures since the temperature dependence of thermal conductivity of all materials constituting workbench is not available. For this reason, thermal conductivity of LDM at lower temperatures 


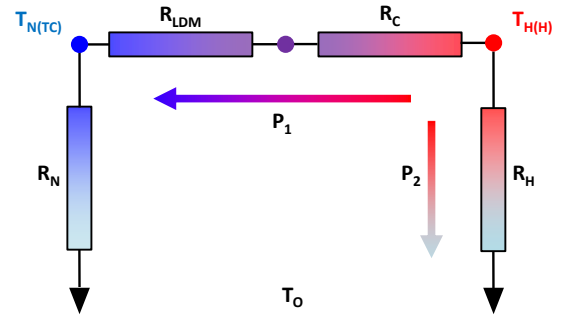

Figure 4: Equivalent thermal circuit of two platforms connected to each other through LDM.

is obtained using an equivalent thermal circuit (Figure 4).

The equivalent thermal circuit was built based on the fact that the heat generated in heater will transfer to the substrate (heat sink) through two paths. One is direct path to bottom of substrate. Another is path through LDM. Considering it, one obtains

$$
P_{H}=P_{1}+P_{2}
$$

where $P_{H}, P_{1}$, and $P_{2}$ are heating power, the amount of heat flowing through LDM, and the amount of heat sinking directly to the bottom of the substrate. Temperature rise of heater of heated platform and thermocouple junction of neighboring platform can be expressed as

$$
\begin{gathered}
\Delta T_{H(H)}=P_{1}\left(R_{L D M}+R_{N}+R_{C}\right)=P_{2} R_{H} \\
\Delta T_{N(T C)}=P_{1} R_{N}
\end{gathered}
$$

where $\Delta T_{H(H)}, \Delta T_{N(T C)}, R_{L D M}, R_{N}, R_{H}$ are the temperature rise of heater of heated platform, temperature rise of thermocouple junction of neighboring platform, thermal resistance of LDM, thermal resistance from thermocouple junction of neighboring platform to bottom of substrate, thermal resistance from heater of heated platform to bottom of substrate, respectively. $R_{C}$ represents sum of thermal resistance from heater to LDM and from LDM to thermocouple junction. Using equation (4) and (6), equation (5) can be reorganized to

$$
R_{L D M}=\frac{P_{H}}{\Delta T_{N(T C)}} R_{H} R_{N}-R_{H}-R_{N}-R_{C}
$$

$R_{H}, R_{N}$, and $R_{C}$ at $300 \mathrm{~K}$ are extracted by FEM. However, we still need to know how these change with temperature for calculating $R_{L D M}$ at various temperatures. In addition, temperature rise of thermocouple junction of neighboring platform produced by heat flow through substrate from heated platform needs to be subtracted from measured $\Delta T_{N(T C)}$ for accurate measurement. These could be investigated using platforms on which LDM is not anchored. The voltage was applied to heater and heating power and temperature rise of thermocouple junctions of heated and neighboring platforms was measured at various temperatures. We could then acquire temperature dependence of thermal resistance from platform tip to heat sink by dividing temperature rise of thermocouple junction by heating power. $R_{H}$ and $R_{N}$ should have same tendency with it even if there might be a small variation in magnitude caused by distance between heater and thermocouple junction. Considering that thermal resistance from platform tip to heat sink mainly originates from thermal resistance of suspended part of platform, it is also reasonable to assume that $R_{C}$ also has same temperature dependence. And, from this measurement, we also obtained the ratio of temperature rise of thermocouple junction of neighboring platform to that of heated platform, which is used to subtract the temperature rise produced by heat transfer through substrate from measured $\Delta T_{N(T C)}$. We found that the amount of heat transferred through substrate is equivalent to that through LDM having thermal conductance of $\sim 1 \times 10^{-8} \mathrm{~W} / \mathrm{K}$. Therefore, if the thermal conductance of LDM is comparable to or smaller than this value, the parasitic heat flow will reduce the accuracy of the measurement of the thermal conductivity of LDM.

\section{Seebeck Coefficient}

Seebeck coefficient of LDM can be calculated by

$$
S_{L D M}-S_{P t}=\frac{V_{T E}}{\Delta T_{H(T C)}-\Delta T_{N(T C)}}
$$

where $S_{L D M}, S_{P t}, V_{T E}$, and $\Delta T_{H(T C)}$ are Seebeck coefficient of LDM, Seebeck coefficient of platinum, and measured thermoelectric voltage, and temperature rise of thermocouple junction of heated platform, respectively. In most cases, $S_{P t}$ is negligibly small compared to $S_{L D M}$ because we are interested in material which has high Seebeck coefficient. However, in case that $S_{L D M}$ is small, $S_{P t}$ needs to be added to the measured Seebeck coefficient. Also, there might be a temperature difference between thermocouple junction and end of LDM if they are positioned far from each other or thermal conductance of LDM is large. Then, instead of $\Delta T_{H(T C)}-\Delta T_{N(T C)}$, we can use temperature difference between two ends of LDM calculated as

$$
\Delta T=P_{1} R_{L D M}=\Delta T_{N(T C)} \frac{R_{L D M}}{R_{N}}
$$

\section{Electrical Conductivity}

Electrical conductivity is measured using two platinum layers connected to each other through LDM. However, resistance of platinum layer should be subtracted from measured resistance. One method is to calculate resistance of platinum layer by using the resistivity measured with calibration structure (inset, Figure 3(a)). Another method is to electrically connect platform tip with one right next to it using FIBID and measure the resistance of the platinum structures. In addition, two probe measurements can also have errors arising from contact resistance but in the case of highly conductive LDMs this is not such a major source of inaccuracy.

\section{Thermoelectric Properties of GaAs/MnAs Core/shell Naowires}

We investigated thermoelectric properties of Gallium Arsenide (GaAs)/Manganese Arsenide (MnAs) core/shell nanowire using the workbench. GaAs/MnAs core/shell nanowire is a suitable material for investigating magneto-thermoelectric effects, such as magneto-caloric, magneto-Seebeck, and spin-Seebeck effects, of quasi-one-dimensional system because MnAs is a ferromagnetic material whose Curie temperature is near room temperature. As a preliminary experiment for magneto-thermoelectric effects, we measured thermoelectric properties as a function of temperature. The nanowire was grown by molecular beam epitaxy and, according to TEM analysis, cross-section of nanowire is a hexagon and the longest diagonal of GaAs core and the thickness of MnAs shell are estimated to be $200 \mathrm{~nm}$ and $15 \mathrm{~nm}$, respectively. IPA solution containing nanowires was drop cast on workbench and FIBID of

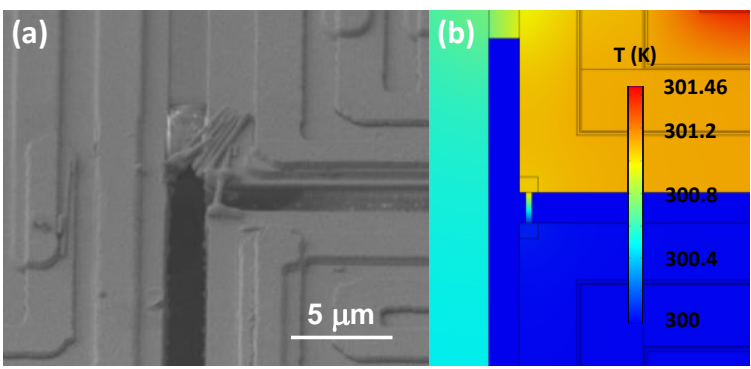

Figure 5: (a) SEM images of workbench on which GaAs/MnAs core/shell nanowire is anchored (b) Color plot of temperature distribution at $300 \mathrm{~K}$ (COMSOL finite element model simulation). 
(a)

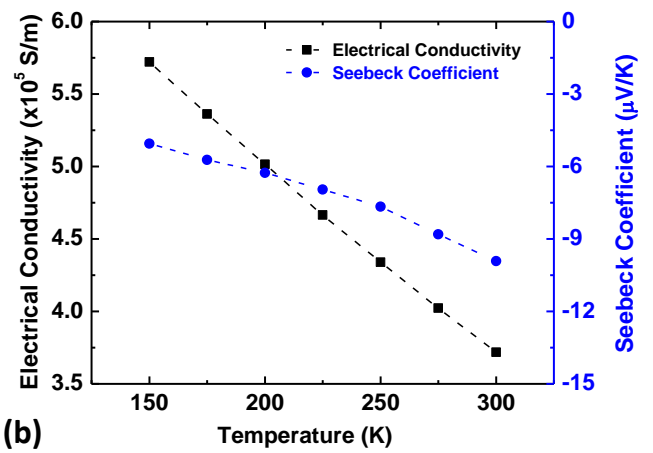

(b)

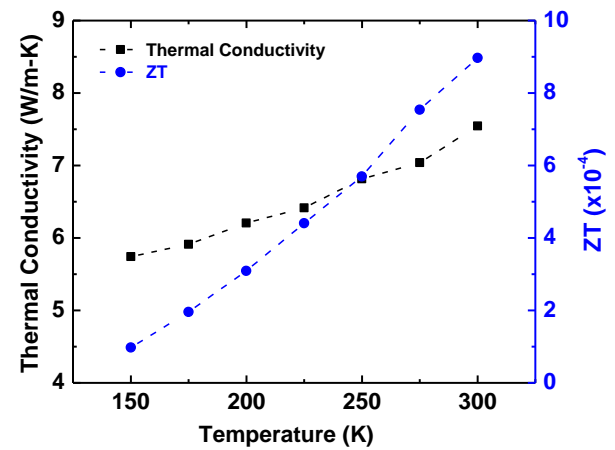

Figure 6: (a) Electrical conductivity of MnAs shell, Seebeck coefficient of nanowire-platinum thermoelectric circuit (b) Thermal conductivity and ZT of nanowire as a function of temperature.

platinum was used to make mechanical contact and improve electrical and thermal contact between nanowire and platinum pad. Figure 5(a) is SEM image of workbench on which single GaAs/MnAs core/shell nanowire is anchored and Figure 5(b) shows temperature distribution of the sample that is FEM simulation result.

Figure 6(a) shows temperature dependence of electrical conductivity of MnAs shell and Seebeck coefficient of nanowire-platinum thermoelectric circuit. The electrical transport (electrical conductivity and Seebeck coefficient) of nanowire are dominated by $\mathrm{MnAs}$ shell because GaAs core is intrinsic. Considering Seebeck coefficient of platinum is $-5 \mu \mathrm{V} / \mathrm{K}$ [10], absolute Seebeck coefficient of MnAs shell is $-16.9 \mu \mathrm{V} / \mathrm{K}$ at $300 \mathrm{~K}$ which is larger than previously reported Seebeck coefficient of bulk MnAs $(-13 \mu \mathrm{V} / \mathrm{K})$ [11]. It might be explained by quantum size effect on Seebeck coefficient.

Figure 6(b) shows the temperature dependence of thermal conductivity and thermoelectric figure of merit ZT of the nanowire. In contrast with electrical transport, most part of thermal transport occurs through the GaAs core because of its large cross-sectional area and dominant lattice thermal conductivity. The thermal conductivity of nanowire is $7.5 \mathrm{~W} / \mathrm{m}-\mathrm{K}$ at $300 \mathrm{~K}$. It is smaller than theoretical value $(20 \mathrm{~W} / \mathrm{m}-\mathrm{K})$ of $200 \mathrm{~nm}$-diameter GaAs nanowire which is recently reported [12]. It decreases with decreasing temperature whereas theoretical value has a maximum value near $100 \mathrm{~K}$. These are thought to be because GaAs core has stacking faults perpendicular to the wire axis. In some regions, the nanowires undergo a transformation in crystal structure from zinc blende with a [111] growth direction to wurtzite with a [0001] growth direction [13]. It would increase phonon-boundary scattering and lead to smaller thermal conductivity than the theoretical value. Furthermore, it would make phonon-boundary scattering more dominant than Umklapp phonon-phonon scattering that results in the increasing thermal conductivity with decreasing temperature. For calculating ZT, the Seebeck coefficient of platinum [12] was added to the measured Seebeck coefficient of nanowire-platinum thermoelectric circuit. Also, the electrical conductivity was recalculated with dimension of core and shell instead of only shell because we need electrical conductivity of core/shell nanowire instead of that of shell for obtaining ZT.

\section{ACKNOWLEDGEMENT}

This work was supported by the NSF under MRSEC Grant DMR-0820404. The authors acknowledge use of facilities at the PSU site of NSF NNIN. Travel support has been generously provided by the Transducer Research Foundation.

\section{REFERENCES}

[1] M. Dresselhaus, G. Dresselhaus, X. Sun, Z. Zhang, S. Cronin, and T. Koga, "Low-dimensional thermoelectric materials," Physics of the Solid State, vol. 41, pp. 679-682, 1999.

[2] A. I. Hochbaum, R. Chen, R. D. Delgado, W. Liang, E. C. Garnett, M. Najarian, A. Majumdar, and P. Yang, "Enhanced thermoelectric performance of rough silicon nanowires," Nature, vol. 451, pp. 163-167, 2008.

[3] L. Shi, D. LI, C. Yu, W. Jang, D. Kim, Z. Yao, P. Kim, and A. Majumdar, "Measuring thermal and thermoelectric properties of one-dimensional nanostructures using a microfabricated device," Journal of heat transfer, vol. 125, pp. 881-888, 2003.

[4] A. I. Boukai, Y. Bunimovich, J. Tahir-Kheli, J. K. Yu, W. A. Goddard Iii, and J. R. Heath, "Silicon nanowires as efficient thermoelectric materials," Nature, vol. 451, pp. 168-171, 2008.

[5] M. A. Panzer, M. Shandalov, J. A. Rowlette, Y. Oshima, Y. W. Chen, P. C. McIntyre, and K. E. Goodson, "Thermal properties of ultrathin hafnium oxide gate dielectric films," Electron Device Letters, IEEE, vol. 30, pp. 1269-1271, 2009.

[6] Y. Sungtaek Ju, X. REN, W. XIAO, N. Smith, R. Fontana, W. Lee, K. Carey, M. Ho, D. Hsiao, and B. Gurney, "A combined experimental and numerical study of temperature rise in GMR sensors due to self-heating," IEEE transactions on magnetics, vol. 37, pp. 1701-1703, 2001.

[7] C. H. Mastrangelo, Y.-C. Tai, and R. S. Muller, "Thermophysical properties of low-residual stress, silicon-rich, LPCVD silicon nitride films," Sensors and Actuators A: Physical, vol. 23, pp. 856-860, 1990.

[8] M. B. Kleiner, S. A. Kuhn, and W. Weber, "Thermal conductivity measurements of thin silicon dioxide films in integrated circuits," Electron Devices, IEEE Transactions on, vol. 43, pp. 1602-1609, 1996.

[9] A. D. McConnell, S. Uma, and K. E. Goodson, "Thermal conductivity of doped polysilicon layers," Microelectromechanical Systems, Journal of, vol. 10, pp. 360-369, 2001.

[10] R. Huebener, "Size effect on phonon drag in platinum," Physical Review, vol. 140, p. A1834, 1965.

[11] U. Neitzel and K. Barner, "The thermoelectric power of MnAs1-xPx compounds," Journal of Physics C: Solid State Physics, vol. 11, p. 4975, 1978.

[12] P. N. Martin, Z. Aksamija, E. Pop, and U. Ravaioli, "Reduced thermal conductivity in nanoengineered rough ge and gaas nanowires," Nano letters, vol. 10, pp. 1120-1124, 2010.

[13] N. Dellas, J. Liang, B. Cooley, N. Samarth, and S. Mohney, "Electron microscopy of GaAs/MnAs core/shell nanowires," Applied Physics Letters, vol. 97, p. 072505, 2010.

\section{CONTACT}

*Srinivas Tadigadapa, sat10@ psu.edu 